\title{
A Novel Multiparametric Approach for Analysis of Cytoplasmic Immunoglobulin Light Chains by Flow Cytometry
}

Chung-Che Chang, M.D., Ph.D., Bernard C. Schur, M.T., Bal. Kampalath, M.D., Paul Lindholm, M.D., Carl G. Becker, M.D., David H. Vesole, M.D., Ph.D.

Departments of Pathology (CCC, BCS, BK, PL, CGB) and Medicine (DHV), Medical College of Wisconsin, Milwaukee, Wisconsin

We describe a novel flow cytometric approach using a two-step acquisition technique to determine the cytoplasmic immunoglobulin light chains (LC) expression. Samples were prepared by a lysed-wholeblood technique and incubated with CD38-PE (phycoerythrin) and CD45-FITC (fluorescein isothiocyanate). The cells were fixed and acquired on an FACSCalibur flow cytometer (first acquisition). The cells were then permeabilized, incubated with either kappa-FITC or lambda-FITC and reacquired (second acquisition). Analysis of the data was performed by gating on the differing intensities of CD38 and evaluating them for the presence of a shifting FITC-positive population from the first acquisition to the second acquisition. The FITC fluorescence intensity of the second acquisition was equal to the sum of surface CD45 expression obtained during the first acquisition and the cytoplasmic LC expression obtained during the second acquisition. Thus, the shifting (increase) of FITC fluorescence intensity during the second acquisition is specifically due to the cytoplasmic expression of either the kappa or lambda LC. We studied 15 multiple myeloma (MM) patients and 10 controls (samples from patients without plasma cell dyscrasias). None of the controls showed evidence of any clonal populations. Thirteen of 15 MM patients exhibited clonal plasma cells (CD38 bright), ranging from $0.01 \%$ to $34 \%$ of total events collected. In addition, we identified another minute clonal population of lymphocytes (CD38 dim, CD45 bright, low forward and side scatter) in 12 of $13 \mathrm{MM}$ patients with clonal plasma cells. This population, ranging from $0.01 \%$ to $0.6 \%$ of total events collected, had the

Copyright (C) 2001 by The United States and Canadian Academy of Pathology, Inc.

VOL. 14, NO. 9, P. 1015, 2001 Printed in the U.S.A.

Date of acceptance: May 25, 2001.

Address reprint requests to: Dr. Chung-Che Chang, Department of Pathology, Medical College of Wisconsin, 9200 W. Wisconsin Avenue, Milwaukee, WI 53226; e-mail: jeffchang@pol.net; fax: 414-805-8444. same LC restriction as the clonal plasma cells. Patients with a ratio of minor clonal population to clonal plasma cells less than 0.07 tended to remain in partial or complete remission than those with a ratio $\geq 0.07\left(4 / 5\right.$ versus $\left.1 / 4, P<.1, \chi^{2}\right)$. We conclude that this method is highly sensitive and permits us to identify the minute clonal population of lymphocytes in MM patients. Our preliminary observations with a small cohort of patients imply that this minute clonal population may have important prognostic significance. The prognostic significance should be confirmed by further studies.

KEY WORDS: Cytoplasmic light chains, Flow cytometry, Two-step acquisition.

Mod Pathol 2001;14(9):1015-1021

Accurate determination of cytoplasmic immunoglobulin (cIg) light chain (LC) expression is important for differentiating reactive plasmacytosis from a clonal plasma cell dyscrasia. The former is caused by polyclonal proliferation of plasma cells; the latter results from clonal proliferation of plasma cells showing LC restriction. Currently, there are several methods available for measuring cIg LC, such as immunohistochemical stain, in situ hybridization for LC mRNA, flow cytometry (FCM) and immunofluorescent stain.

Flow cytometric evaluation of cIg LC has many theoretical advantages over the other methods mentioned above, including simultaneous multiparametric analysis of a large number of cells, computer-aided methods of quantification, and comparatively rapid results. However, routine clinical flow cytometric determination of cIg LC in plasma cells has met with a number of obstacles. Initial flow cytometric methods for detection of intracellular (cytoplasmic/nuclear) antigens in lymphoplasmacytic cells were acknowledged as laborious, technically difficult and not reliably reproducible (1-3). Recently, a variety of permeabilizing agents, such as Triton X-100 (Sigma, St 
Louis, MO), Permafix (Ortho Diagnostic Systems, Raritan, NJ) and FACS lysing solution (Becton Dickinson Immunocytometry Systems, San Jose, CA) have enabled access to intracellular antigens without severely altering important light-scattering properties $(2,4-6)$. These methods have somewhat improved the accuracy of cytoplasmic cIg LC determination by FCM.

Even with these newer and more sensitive techniques, the interpretation of results can sometimes remain very difficult, particularly when the number of plasma cells is small. Thus, determination of cIg LC by FCM has not been commonly used in clinical FCM laboratories.

To improve the measurement of cIg LC by FCM, we have developed a novel flow cytometric approach using a two-step acquisition technique. This method is highly sensitive with the ability to detect clonal plasma cells numbering as few as $0.01 \%$ of total events collected. This sensitivity is in the range of minimal residual disease detection. Additionally, this method permits identification of a minute clonal population of lymphocytes expressing cIg LC. The results of this pilot study imply that this population might have important prognostic significance for patients with multiple myeloma (MM). Further studies are indicated to confirm our observation.

\section{MATERIALS AND METHODS}

\section{Patients}

Twenty-five samples, 5 peripheral blood and 20 bone marrow, were obtained from $15 \mathrm{MM}$ patients (Cases 1 through 15, Table 1) and 10 control patients (Cases 16 through 25, Table 1). The 10 control patients had marrow examinations for hematologic disorders other than plasma cell dyscrasias, including myelodysplasia, anemia and thrombocytopenia. The MM patients studied included patients at variable stages of disease including newly diagnosed, complete or partial clinical remission, and progressive disease.

\section{FCM with Two-Step Acquisition}

Bone marrow and peripheral blood specimens, anticoagulated with either EDTA or heparin, were processed using a lysed-whole blood technique with ammonium chloride as a lysing agent. Samples were then washed and resuspended with RPMI buffer (Gibco, Grand Island, NY) to a concentration of approximately $2 \times 10^{6}$ cells per $\mathrm{mL}$.

Cells from each sample were placed into two tubes. Both tubes then incubated with phycoerythrin (PE)-coupled anti-CD38 and fluorescein isothiocyanate (FITC)-coupled anti-CD45 (Becton Dickenson) at 20 to $25^{\circ} \mathrm{C}$ for 15 minutes in the dark.

TABLE 1. The Results of Cytoplasmic Immunoglobulin Light Chain Expression by Flow Cytometry Using Two-Step or One-Step Procedures and by Immunofixation

\begin{tabular}{|c|c|c|c|c|c|c|c|c|}
\hline \multirow{2}{*}{ Case \# } & \multirow{2}{*}{ Specimen } & \multirow{2}{*}{ LCR } & \multicolumn{2}{|c|}{ PC\% } & \multicolumn{2}{|c|}{ K/L Ratio } & \multirow{2}{*}{ CL\% } & \multirow{2}{*}{ IFE } \\
\hline & & & (Two-step) & (One-step) & (Two-step) & (One-step) & & \\
\hline 1 & $\mathrm{BM}$ & $\mathrm{K}$ & 7.30 & NA & 381 & NA & 0.32 & NA \\
\hline 2 & $\mathrm{BM}$ & $\mathrm{K}$ & 3.25 & 0.59 & 218 & 12.4 & 0 & No spike present \\
\hline 3 & $\mathrm{BM}$ & K & 1.00 & NA & 18.5 & NA & 0.07 & $\operatorname{IgGK}(4.2 \mathrm{gm} / \mathrm{dL})$ \\
\hline 4 & $\mathrm{BM}$ & $\mathrm{K}$ & 0.06 & NA & 7.8 & NA & 0.01 & NA \\
\hline 5 & $\mathrm{BM}$ & $\mathrm{K}$ & 2.46 & 0.36 & 176 & 27.00 & 0.16 & NA \\
\hline 6 & $\mathrm{BM}$ & $\mathrm{K}$ & 9.50 & NA & 82 & NA & 0.10 & IgGK $(1.6 \mathrm{gm} / \mathrm{dL})$ \\
\hline 7 & $\mathrm{BM}$ & $\mathrm{K}$ & 4.00 & 5.20 & 17.5 & 4.75 & 0.57 & NA \\
\hline 8 & $\mathrm{BM}$ & $\mathrm{L}$ & 1.40 & NA & 0.01 & NA & 0.08 & $\operatorname{IgGL}(5.9 \mathrm{gm} / \mathrm{dL})$ \\
\hline 9 & $\mathrm{BM}$ & $\mathrm{L}$ & 15.20 & 1.09 & 0.002 & 0.01 & 0.30 & NA \\
\hline 10 & $\mathrm{BM}$ & $\mathrm{L}$ & 34.00 & 4.9 & 0.02 & 0.03 & 0.6 & $\begin{array}{c}\text { Free L }(0.9 \mathrm{gm} / \\
\mathrm{dL})\end{array}$ \\
\hline 11 & $\mathrm{BM}$ & $\mathrm{L}$ & 0.74 & 0.12 & 0.05 & 0.58 & 0.07 & NA \\
\hline 12 & $\mathrm{~PB}$ & $\mathrm{~K}$ & 0.09 & NA & 4.5 & NA & 0.06 & IgGK $(1.7 \mathrm{gm} / \mathrm{dL})$ \\
\hline 13 & $\mathrm{~PB}$ & $\mathrm{~K}$ & 0.01 & NA & 7.00 & NA & 0.05 & IgGK $(1.6 \mathrm{gm} / \mathrm{dL})$ \\
\hline 14 & PB & - & 0.1 & NA & 1.8 & NA & 0 & $\begin{array}{l}\text { IgGL (faint band) } \\
\text { IgGK }(0.5 \mathrm{gm} / \mathrm{dL})\end{array}$ \\
\hline 15 & $\mathrm{~PB}$ & - & 0.01 & NA & 1.5 & NA & 0 & NA \\
\hline 16 & $\mathrm{BM}$ & - & 0.18 & 0.02 & 0.58 & 0.75 & 0 & NA \\
\hline 17 & $\mathrm{BM}$ & - & 0.14 & 0.03 & 1.22 & 2.20 & 0 & NA \\
\hline 18 & $\mathrm{BM}$ & - & 0.06 & NA & 0.6 & NA & 0 & NA \\
\hline 19 & $\mathrm{BM}$ & - & 0.05 & NA & 1.50 & NA & 0 & NA \\
\hline 20 & $\mathrm{BM}$ & - & 0.02 & NA & 0.62 & NA & 0 & NA \\
\hline 21 & $\mathrm{BM}$ & - & 1.5 & 0.39 & 1.16 & 0.90 & 0 & NA \\
\hline 22 & $\mathrm{BM}$ & - & 0.03 & NA & 0.67 & NA & 0 & NA \\
\hline 23 & $\mathrm{BM}$ & - & 0.24 & NA & 0.62 & NA & 0 & NA \\
\hline 24 & $\mathrm{BM}$ & - & 0.04 & NA & 0.54 & NA & 0 & NA \\
\hline 25 & $\mathrm{~PB}$ & - & 0.04 & NA & 3.5 & NA & 0 & NA \\
\hline
\end{tabular}

BM, bone marrow; CL, clonal lymphocyte; IFE, immunofixation electrophoresis; Ig, immunoglobulin; K, kappa light chain; L, lambda light chain; LCR, light chain restriction pattern; NA, not available/not applicable; PB, peripheral blood; PC, plasma cell. 
After incubation, the cells were fixed using $150 \mu \mathrm{L}$ of Reagent A (Fix \& Perm Kit, Caltag Laboratories, Burlingame, CA). Next, $3 \mathrm{~mL}$ of phosphate buffered saline with sodium azide was added and samples were centrifuged for 5 minutes at $400 \times g$. The cell pellet was resuspended in $300 \mu \mathrm{L}$ of phosphate buffered saline and 20,000 events were acquired in list mode on an FACSCalibur Flow Cytometer (Becton Dickenson) using CellQuest software (first acquisition).

After the first acquisition, cell membranes were permeabilized by adding $150 \mu \mathrm{L}$ of Reagent B (Fix \& Perm Kit) into the same tubes. After permeabilization, one tube was incubated with FITC-coupled polyclonal anti-kappa LC and the other with FITCcoupled polyclonal anti-lambda LC (DAKO, Carpinteria, CA). After incubation, 50,000 events were collected (second acquisition) from each tube. Analysis of the data was achieved by gating on the differing intensities of CD38 and evaluating them for the presence of a shifting FITC-positive population from the first acquisition to the second acquisition (Figs. 1 and 2).

The background fluorescence level for each specimen was established using cells incubated with appropriate fluorochrome-coupled isotype control antibodies. Fluorochrome compensation was adjusted electronically, utilizing normal peripheral blood leukocytes labeled with FITC-coupled antiCD3 and PE-coupled anti-CD19.

\section{FCM with One-Step Acquisition}

In nine patients (six MM and three controls), the measurement of cIg LC was also performed using the procedure as suggested by the manufacture of the permeabilizing reagent (Fix \& Perm Kit). The cells were fixed with $100 \mu \mathrm{L}$ of Reagent A and then permeabilized with Reagent $B$. The cells were incubated with peridinin chlorophyll protein (PerCP)coupled anti-CD45, PE-coupled anti-CD38 and either FITC-coupled anti-kappa LC or FITC-coupled anti-lambda LC. After the incubation, acquisition of the cells and analysis of the data were performed.

\section{Immunofixation Electrophoresis}

Serum and/or urine immunofixation electrophoresis studies were performed on Sebia HYRYS system (Sebia Electrophoresis, Norcross, GA) following standardized procedures and manufactur-
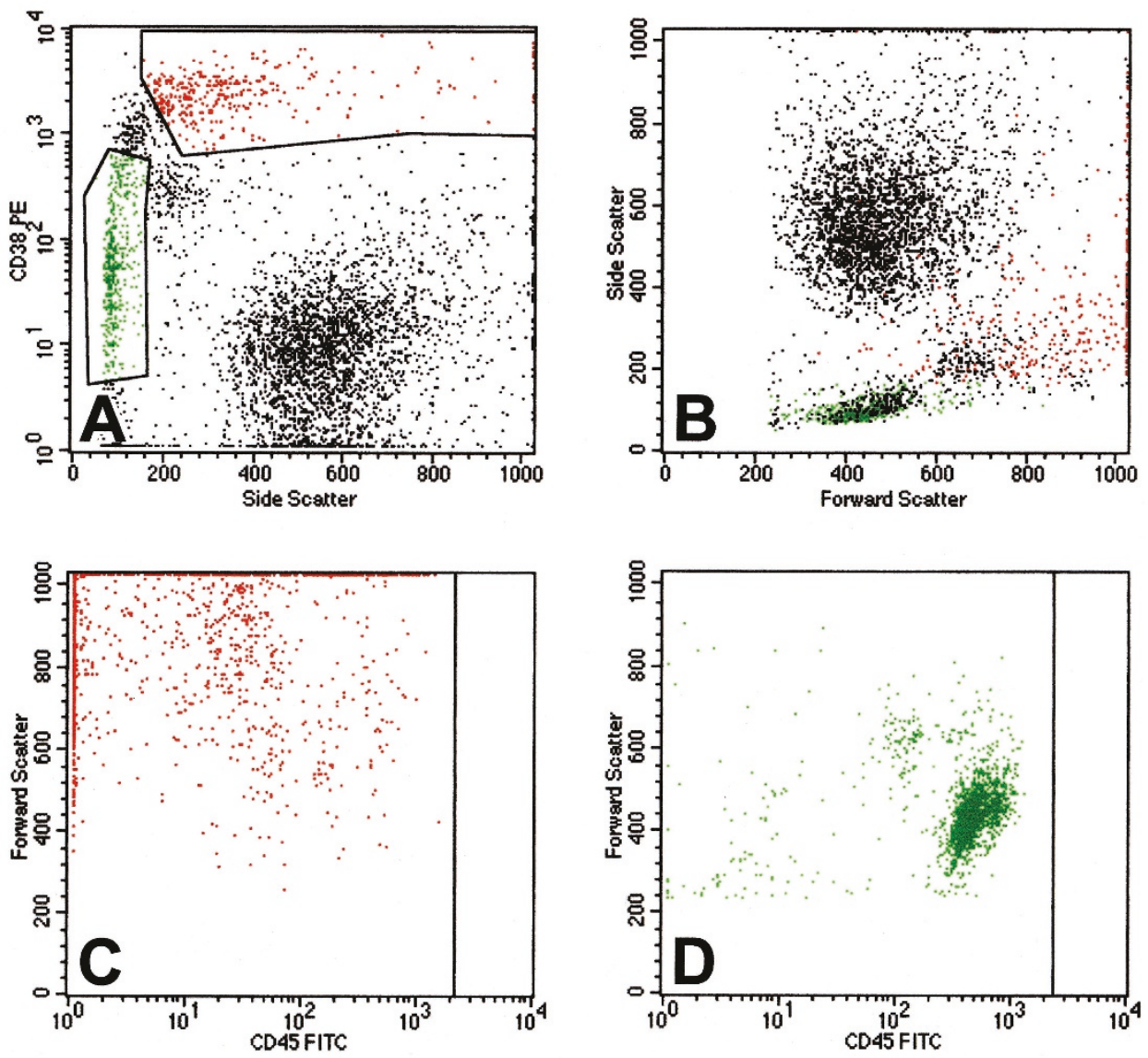

FIGURE 1. Histograms of the first acquisition (before permeabilization). Upper left histogram (A) shows gating of plasma cells in red and lymphocytes in green. The plasma cells are characterized by bright CD38 expression (A) and dim to intermediate CD45 expression (C). The lymphocytes are characterized by low forward scatter and side scatter (B), strong CD45 expression (D) and dim CD38 expression (A). 
er's instructions. Eight of the MM patients had serum and/or urine samples available for analysis.

\section{RESULTS}

\section{Two-Step Acquisition Procedure}

The two-step acquisition procedure was designed to visualize the cell population that showed an increase (or shift) of FITC fluorescence intensity during the second acquisition (Figs. 1 and 2). Because the FITC fluorescence intensity of the second acquisition was equal to the sum of surface CD45 expression obtained during the first acquisition and the cIg expression obtained during the second ac- quisition, this increase is specifically due to the cytoplasmic expression of either the kappa or lambda LC. For example, in Fig. 2C, the cells on the right side of the vertical line showing increased FITC fluorescence intensity during the second acquisition (as compared with the first acquisition as in Fig. 1C) were the plasma cells expressing cytoplasmic kappa LC. Similarly, in Fig. 2D, the cells on the right side of the vertical line were the plasma cells expressing cytoplasmic lambda LC.

The LC restriction was defined as a kappa to lambda ratio more than 4.0 (kappa LC restriction) or less than 0.5 (lambda LC restriction) based on commonly used criteria (7-9). None of the 10 controls
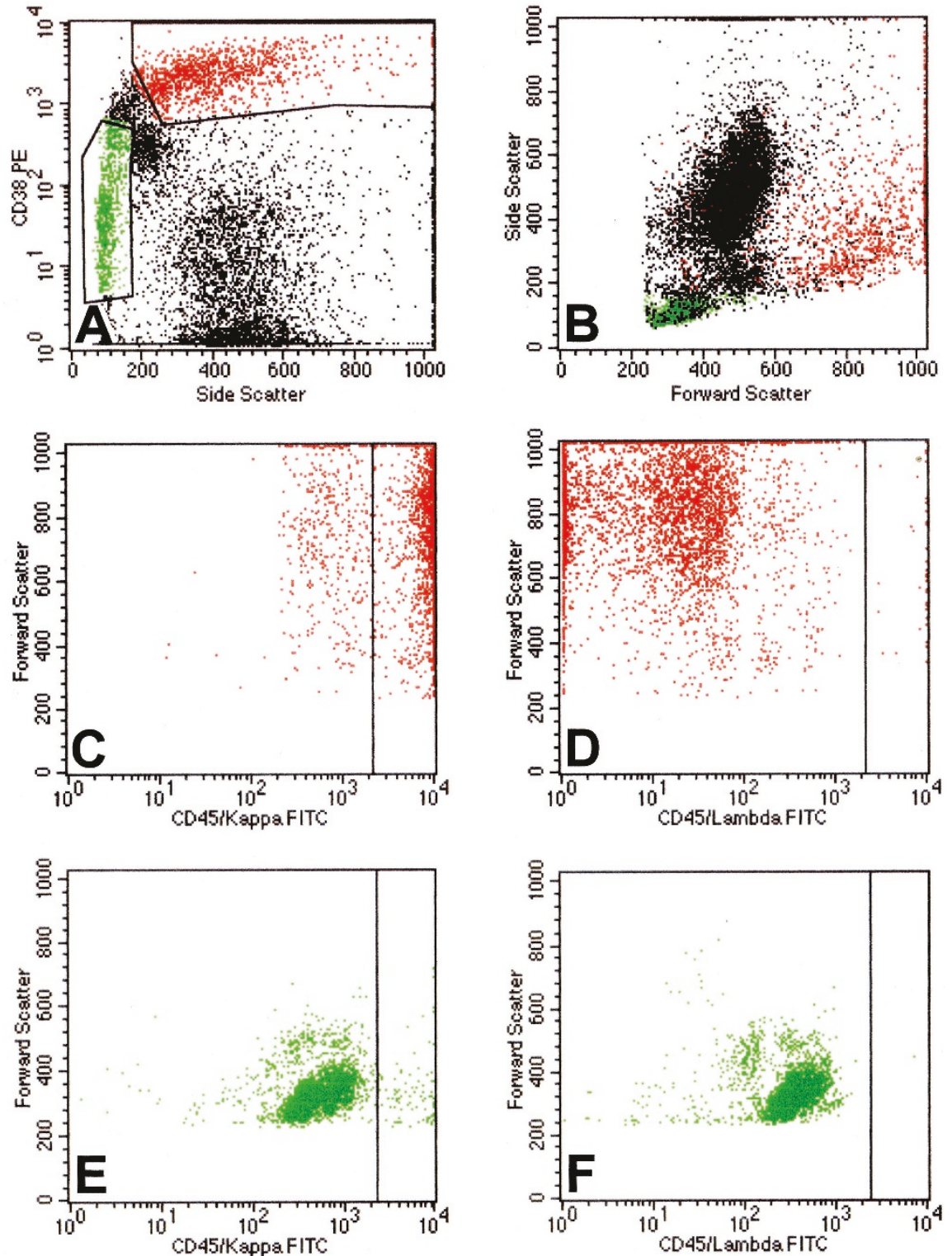

FIGURE 2. Histograms of the second acquisition (after permeabilization). The upper left and upper right histograms (A, B) are as in Figure 1, A-B. The plasma cells express monoclonal cytoplasmic kappa light chain (C, D). Note the shift of FITC fluorescence intensity of the plasma cells as compared with Figure 1C. There is a small population of lymphocytes expressing monoclonal cytoplasmic kappa light chains (E, F as compared with Fig. 1D). These cells may represent malignant B-cells components of MM. The increase in dot intensity from Figure 1 to Figure 2 is due to more events collected in the second acquisition $(50,000)$ than in the first acquisition $(20,000)$. 
showed LC restriction. Thirteen (87\%) of 15 MM patients exhibited clonal plasma cells (CD38 bright), ranging from $0.01 \%$ to $34 \%$ of the total events collected. Except Case 12 (kappa/lambda ratio of 4.5), the remaining $12 \mathrm{MM}$ patients had very clear-cut LC pattern with kappa to lambda ratios well over or well under the defined cutoffs (Table 1). Two patients failed to show monoclonal plasma cells in the peripheral blood. One of them (Case 14) had two clonal peaks of IgG lambda and IgG kappa, by immunofixation electrophoresis. So, the clonality of plasma cells could not be determined by FCM. The other patient (Case 15) was in complete clinical remission.

In 12 of $13 \mathrm{MM}$ patients with monoclonal plasma cells, we identified another minute clonal population of lymphocytes (CD38 dim, CD45 bright, low forward and side scatter). This population had the same LC restriction as the plasma cells (Figs. 1 and 2). These cells were very few in number ranging from $0.01 \%$ to $0.6 \%$ of total events collected and may represent malignant B-cells components of MM.

To evaluate the clinical significance of this minor clonal population, we had treatment response information for nine patients with a median follow-up length of 7 months. Patients with a ratio of minor clonal population to clonal plasma cells less than 0.07 tended to remain in partial or complete remission than those with a ratio $\geq 0.07\left(4 / 5\right.$ versus $1 / 4, P<.1, \chi^{2}$, Table 2). The majority (three-fourths) of patients with a ratio $\geq 0.07$ had progressive disease.

\section{Comparison of the Results by Two-Step Acquisition Procedure and the Results by One-Step Acquisition Procedure}

The LC restriction patterns by two-step acquisition procedure or one-step acquisition procedure were almost identical. However, the two-step acquisition method showed more distinct LC restriction pattern (more skewed kappa to lambda ratios) in plasma cells than the one-step acquisition method (Table 1), particularly when the amount of plasma cells was small. In one case (Case 11), onestep procedure failed to demonstrate LC restriction

TABLE 2. The Ratio of Clonal Lymphocytes (CL) to Neoplastic Plasma Cell (PC) and the Status of Disease in Multiple Myeloma Patients

\begin{tabular}{rcl}
\hline Case $\#^{a}$ & CL/PC Ratio & Current Disease Status \\
\hline 2 & 0 & Complete remission \\
6 & 0.01 & Partial remission \\
10 & 0.02 & Progressive disease \\
1 & 0.04 & Complete remission \\
8 & 0.06 & Partial remission \\
3 & 0.07 & Progressive disease \\
7 & 0.14 & Progressive disease \\
4 & 0.17 & Partial remission \\
12 & 0.7 & Progressive disease \\
\hline
\end{tabular}

${ }^{a}$ Same case numbers as in Table 1.

CL, clonal lymphocyte; PC, plasma cell. but the two-step procedure showed an obvious lambda LC restriction. Additionally, the two-step method generally revealed a higher percentage of plasma cells than the one-step method in the same samples (Table 1). Therefore, the comparison study of the two procedures was stopped and subsequent samples were only studied using the two-step acquisition method.

Comparison of the Results by Two-Step Acquisition Procedure and the Results by Immunofixation Electrophoresis

The LC restriction patterns in plasma cells as determined by FCM using two-step acquisition were identical to those of immunofixation electrophoresis in six of eight patients who had both studies performed. Four patients had IgG kappa monoclonal peaks, ranging from 1.6 to $4.2 \mathrm{gm} / \mathrm{dL}$, and all of them had clonal plasma cells expressing cytoplasmic kappa LC by FCM. One patient had an IgG lambda monoclonal peak (5.9 gm/dL) and another had a monoclonal peak of free lambda chains ( 0.9 $\mathrm{gm} / \mathrm{dL}$ ); both of them had clonal plasma cells expressing cytoplasmic lambda LC by FCM.

In two patients, the results by the two methods showed discordances. One patient (Case 2) had no monoclonal peak in serum and urine by immunofixation electrophoresis but showed clonal plasma cells expressing kappa LC by FCM. The other patient (Case 14) was described as above.

\section{DISCUSSION}

We report a sensitive method for flow cytometric measurement of cIg LC that can clearly demonstrate LC restriction pattern for supporting the diagnosis of clonal proliferation of plasma cells. The LC restriction can be determined with samples containing clonal plasma cells numbering as few as $0.01 \%$ of total events collected. Additionally, this method permits us to detect another minute clonal population of B-lymphocytes in patients with MM. The results of this pilot study imply that patients in whom this minute population of cells were increased tended to have progressive disease.

Our two-step approach has several advantages over the previously reported methods $(4,6)$ that used similar permeabilizing agents with one-step approach. First, the first acquisition of surface markers allow us to clearly define the plasma cell population (bright CD38) without the effect of distortion of light scatter characteristics caused by permeabilization. Second, the amount of cells expressing kappa or lambda LC can be accurately determined by objective observation of the fluorescence intensity shift from the first acquisition to the second acquisition (Figs. 1 and 2). This results in 
much-improved LC restriction patterns that correlate well with those determined by immunofixation electrophoresis. However, this correlation should be validated by further studies, because only eight patients had immunofixation electrophoresis data for analysis. Third, our preliminary observations suggest that expression of other markers, such as CD56 and CD19, of the clonal plasma cells and lymphocytes can be determined using other fluorochromes coupled antibodies (data not shown). This permits the simultaneous analysis of four antigens (i.e., three surface antigens plus one cytoplasmic antigen) expression using a flow cytometer capable of three-color analysis or five antigens (i.e., four surface antigens plus one cytoplasmic antigen) expression using a flow cytometer capable of fourcolor analysis.

Additionally, the sensitivity in detecting clonal plasma cells inpatients with $\mathrm{MM}$ is markedly enhanced by this method to as few as $0.01 \%$ of total events collected. With this sensitivity, the possibility of monitoring minimal residual disease by FCM in MM patients can be further pursued using peripheral blood samples, which are easier to obtain and less invasive than bone marrow samples. Our preliminary results show that clonal plasma cells were present in peripheral blood from two MM patients (Cases 12 and 13) at the amount of $0.06 \%$ and $0.05 \%$ of the total events collected, respectively.

By combining surface marker analysis using CD38 and CD45 and cytoplasmic LC determination, we have detected other clonal lymphocytes in addition to the clonal plasma cells in-patients with MM. These lymphocytes (as defined by expressing dim surface CD38 and strong surface CD45), which have monoclonal cIg LC identical to that of clonal plasma cells, are present not only in peripheral blood, as observed by others (10-15), but also in the bone marrow of MM patients. Usage of additional markers including CD19, CD20 and CD56 to further characterize the clonal plasma cells and clonal lymphocytes is currently under development.

The clinical significance of the clonal B-lymphocytes remains uncertain. It has been implied that the presence of clonal B-lymphocytes, which are not eradicated by chemotherapy, is the main cause of the incurability in MM $(11-13,16)$. Neoplastic plasma cells in MM are usually depleted by chemotherapy, but recent studies have shown that the clonal B-lymphocytes survive despite intensive chemotherapy (10-12). In vitro, these cells express multidrug resistance protein (P-glycoprotein) encoded by MDR1 gene and exhibit a very efficient drug export capacity leading to resistance to multiple chemotherapeutic agents $(10,12,16)$. The clonal B-cells, which have the same IgH gene rearrangements as the bone marrow neoplastic plasma cells, have been suggested to be members of the malignant clone in my- eloma. Our preliminary results with a small cohort of patients imply that the ratio of clonal lymphocytes to neoplastic plasma cells may correlate with clinical course. In this pilot study, the patients with ratio more than 0.07 tends to appear more likely to have progressive disease than those with ratio less than 0.07 .

In summary, our results indicate that this novel procedure for determining cIg LC expression is highly sensitive with the ability to detect clonal plasma cells numbering as few as $0.01 \%$ of total events collected. Further study to evaluate the clinical significance of the monoclonal lymphocyte population identified by this method in MM patients, which may represent malignant B-cells components, is indicated.

Acknowledgments: We thank Dr. Ronald P. Cleveland, MetroHealth Medical Center, Case Western Reserve University, Cleveland, Ohio for his critical review of the manuscript, Joshua Johnson, Dynacare Laboratories, Milwaukee, Wisconsin for technical assistance and Mary Fazio for her help in collecting clinical samples.

\section{REFERENCES}

1. Bardales RH, Al-Katib AM, Carrato A, Koziner B. Detection of intracytoplasmic immunoglobulin by flow cytometry in B-cell malignancies. J Histochem Cytochem 1989;37:83-9.

2. Barlogie B, Alexanian R, Pershouse M, Smallwood L, Smith L. Cytoplasmic immunoglobulin content in multiple myeloma. J Clin Invest 1985;76:765-9.

3. Slaper-Cortenbach IC, Admiraal LG, Kerr JM, van Leeuwen EF, von dem Borne AE, Tetteroo PA. Flow cytometric detection of terminal deoxynucleotidyl transferase and other intracellular antigens in combination with membrane antigens in accurate lymphoblastic leukemias. Blood 1988;72:163944

4. Shaw GR, Gonering E, Koller S. Techniques to improve flow cytometric detection of light chain restriction. Clin Lab Sci 1996;9:292-7.

5. Perry A, Duenzl ML, Ansari MQ. Flow cytometric terminal deoxynucleotidyl transferase analysis: evaluation of Triton X-100 and methanol permeabilization methods compared with immunofluorescence microscopy. Arch Pathol Lab Med 1994;118:1119-22.

6. Groeneveld K, te Marvelde JG, van den Beemd MW, Hooijkaas H, van Dongen JJ. Flow cytometric detection of intracellular antigens for immunophenotyping of normal and malignant leukocytes. Leukemia 1996;10:1383-89.

7. Sobol RE, Dillman RO, Collins H, Griffiths JC, Green MR, Royston I. Applications and limitations of peripheral blood lymphocyte immunoglobulin light chain analysis in the evaluation of non-Hodgkins lymphoma. Cancer 1985;56:200510.

8. Hsu SM, Cossman J, Jaffe ES. Lymphocyte subsets in normal human lymphoid tissues. Am J Clin Pathol 1983;80:21-30.

9. Witzig TE, Li CY, Tefferi A, Katzmann JA. Measurement of the intensity of cell surface antigen expression in B-cell chronic lymphocytic leukemia. Am J Clin Pathol 1994;101:312-7.

10. Szczepek AJ, Seeberger K, Wizniak J, Mant MJ, Belch AR, Pilarski LM. A high frequency of circulating B cells share clonotypic Ig heavy-chain VDJ rearrangements with autologous bone marrow plasma cells in multiple myeloma, as 
measured by single-cell and in situ reverse transcriptasepolymerase chain reaction. Blood 1998;92:2844-55.

11. Pilarski LM, Mant MJ, Belch AR. Drug resistance in multiple myeloma: novel therapeutic targets within the malignant clone. Leuk Lymphoma 1999;32:199-210.

12. Pilarski LM, Belch AR. Circulating monoclonal B cells expressing $\mathrm{P}$ glycoprotein may be a reservoir of multidrugresistant disease in multiple myeloma. Blood 1994;83:724-36.

13. Knauf WU, Pochanke G, Ho AD. Detection of circulating monoclonal lymphocytes in multiple myeloma patients by analysis of gene rearrangements: correlation with progressive disease. Leuk Res 1993;17:341-5.
14. Billadeau D, Quam L, Thomas W, Kay N, Greipp P, Kyle R, et al. Detection and quantification of malignant cells in the peripheral blood of multiple myeloma patients. Blood 1992; 80:1818-24.

15. Bergsagel PL, Smith AM, Szczepek A, Mant MJ, Belch AR, Pilarski LM. In multiple myeloma, clonotypic B-lymphocytes are detectable among CD19+ peripheral blood cells expressing CD38, CD56, and monotypic Ig light chain. Blood 1995; 85:436-47.

16. Pilarski LM, Belch AR. Intrinsic expression of the multidrug transporter, P-glycoprotein 170, in multiple myeloma: implications for treatment. Leuk Lymphoma 1995;17:367-74.

\section{Book Review}

\section{Zimmerman, RA, Gibby, WA, Carmody, RF, ed- itors: Neuroimaging: Clinical and Physical Principles, 1656 pp, New York, Springer- Verlag, 2000 (\$195.00).}

This is a carefully constructed monumental resource for neuroradiologists, neurosurgeons, neuropathologists, neurologists, and all other physicians interested in neurosciences. Bulky by design but very user-friendly, it is encyclopedic and relies on a well-thought-through hierarchical search engine for locating topics of interest. It compares very favorably with Anne Osborn's Diagnostic Radiology but also has additional most useful features critical for understanding neuroimaging today.

The table of contents provides chapter headings under each of six main parts. A chapterspecific table of contents is supplied at the beginning of each chapter, which more accurately directs the researcher to a desired topic. Combined with the index found at the end, this construct provides an almost complete directory at all points in the text. The text is comprehensive yet succinct. It is wonderfully illustrated with "Aunt-Minnie" radiologic studies and simple schematics. At times it seems as if the figures dwarf the text, which is completely acceptable in this visually oriented discipline.

The volume fully describes and illustrates the field of nuclear magnetic resonance imaging, from the basic principles of obtaining images by "sampling. . .the energy of processing nuclear dipoles" (p. 73), to the exceedingly complex principles behind obtaining a FLAIR (FLuid Attenu- ated Inversion Recovery) sequence of images and predicting a given material's signal intensity. The authors successfully correlate MR physics and principles with clinical neuroscience. Each of the currently used MR techniques is discussed in this single volume.

The book is comprehensive, but still some potentially important topics are missing. For example, Charcot-Marie-Tooth disease, a cause of diffuse nerve root enlargement, is not addressed. Colpocephaly, an abnormal enlargement of the occipital horns of the lateral ventricles seen commonly in cerebral palsy, either was absent or well hidden so that I could not find it. Finally, trigeminal neuralgia or tic douloureux, which often is associated with dolichoectasia of the basilar artery, is also neglected. There are also some minor spelling errors. For example, "the rhabdomyoma... is begnign" (page 681), whereas on page 1303 the reader will find "the main season [reason] myelograms are still performed." Finally, "Two neoplasms known to cause oncotic aneurysms are left arterial [atrial] myxoma and choriocarcinoma" on page 826. These minor deficiencies, however, should not detract one from recognizing this wonderful book for what it truly is-a major new contribution to neurosciences. I assume that it will be widely used as a textbook and reference.

Kyle A. Krehbiel

The University of Kansas School of Medicine Kansas City, Kansas 